\title{
Medications and dosages used in medical assistance in dying: a cross-sectional study
}

\author{
Igor Stukalin MD, Oluwatobi R. Olaiya MD MSc, Viren Naik MD MEd, Ellen Wiebe MD, \\ Mike Kekewich MA, Michaela Kelly MD, Laura Wilding RN, Roxanne Halko RN MPH, \\ Simon Oczkowski MD MHSc
}

\section{Abstract}

Background: There is little evidence describing the technical aspects of medical assistance in dying (MAiD) in Canada, such as medications, dosages and complications. Our objective was to describe clinical practice in providing MAiD in Ontario and Vancouver, Canada, and explore relations between medications used, time until death and complications.

Methods: We conducted a retrospective cohort study of a sample of adult (age $\geq 18 \mathrm{yr}$ ) patients who received MAiD in Ontario between 2016 and 2018, and patients who received MAiD in 1 of 3 Canadian academic hospitals (in Hamilton and Ottawa, Ontario, and Vancouver, British Colombia) between 2019 and 2020. We used de-identified data for 2016-2018 from the Office of the Chief Coroner for Ontario MAiD Database and chart review data for 2019-2020 from the 3 centres. We used multivariable parametric survival analysis to identify relations between medications, dosages and time from procedure start until death.

Results: The sample included 3557 patients (1786 men [50.2\%] and 1770 women [49.8\%] with a mean age of 74 [standard deviation 13] yr). The majority of patients (2519 [70.8\%]) had a diagnosis of cancer. The medications most often used were propofol (3504 cases [98.5\%]), midazolam (3251 [91.4\%]) and rocuronium (3228 [90.8\%]). The median time from the first injection until death was 9 (interquartile range 6) minutes. Standard-dose lidocaine (40-60 mg) and high-dose propofol (> $1000 \mathrm{mg})$ were associated with prolonged time until death (prolonged by a median of $1 \mathrm{~min}$ and $3 \mathrm{~min}$, respectively). Complications occurred in $41 \mathrm{cases}(1.2 \%)$, mostly related to venous access or need for administration of a second medication.

Interpretation: In a large sample of patients who died with medical assistance, certain medications were associated with small differences in time from injection to death, and complications were rare. More research is needed to identify the medication protocols that predict outcomes consistent with patient and family expectations for a medically assisted death.

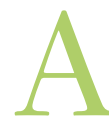

growing number of countries have decriminalized medical assistance in dying (MAiD) as a means for patients to avoid prolonged suffering. ${ }^{1-4}$ Although each jurisdiction's legislative criteria allowing the intentional use of lethal drugs by a clinician vary, in practice, the act of hastening a patient's death can be done in 1 of 2 ways: health care providers can administer a lethal medication directly, or they can prescribe a lethal medication to a patient for self-administration; these 2 methods can also be combined. ${ }^{5}$ In Belgium, the Netherlands and Luxembourg, both clinician-administered and self-administered methods are allowed; Colombia allows only clinician-administered methods; and Switzerland, the Australian state of Victoria and several US states permit self-administration only. ${ }^{5-8}$ In Canada, Bill C-14 defined a process and criteria under which MAiD would be permitted, but it did not specify which methods of hastening death could be used. ${ }^{8}$ As a result, MAiD in Canada includes both administration of lethal medications by physicians and nurse practitioners, and prescription of lethal medications for self-administration (except in Quebec).
Although nearly 7000 Canadians have died with medical assistance, ${ }^{9}$ there is little information on the technical aspects of providing MAiD. The current literature predominantly explores the ethical issues, eligibility, and the impact on patients, families and health care providers. ${ }^{2,10,11}$ However, it is clear that, across Canada, self-administered MAiD is rare, with fewer than 7 cases reported, ${ }^{10}$ a finding consisted with other jurisdictions where both clinician- and self-administered MAiD are legal. ${ }^{1}$ Although the Canadian Association of MAiD Assessors and Providers has released a guidance document for intravenous administration of drugs, ${ }^{12}$ limited data exist on the specific medications, dosages, timing of administration and complications during clinician-administered MAiD.

\section{Competing interests: None declared.}

This article has been peer reviewed.

Correspondence to: Simon Oczkowski, oczkowsj@mcmaster.ca

CMAJ Open 2022 January 18. DOI:10.9778/cmajo.20200268 
The Canadian Association of MAiD Assessors and Providers recommends fixed dosing of midazolam (an anxiolytic), $10 \mathrm{mg}$; propofol (an anesthetic coma-inducing agent), $1000 \mathrm{mg}$; and rocuronium, $200 \mathrm{mg}$, or cisatracurium, $40 \mathrm{mg}$ (neuromuscular blockers to stop respiration). ${ }^{12}$ A scoping review showed that, although virtually all Canadian MAiD protocols use kits with fixed dosages of medications, including an anesthetic (propofol or phenobarbital) and a paralytic, along with a secondary "backup kit" in case of failure, there are variations in the inclusion of anxiolytics (e.g., midazolam), analgesia (e.g., lidocaine, magnesium sulfate) and cardiotoxic medications (e.g., bupivacaine, potassium chloride). ${ }^{1}$ These variations in choice of medication and administration technique may play an important role in ensuring a comfortable and dignified death.

Complications reported from jurisdictions outside Canada include difficulty obtaining intravenous access, longer than expected time to death, pain on injection and need for a second MAiD medication kit owing to technical failure of the first kit. ${ }^{1,13}$ These reports indicate that complications during medication administration can cause further patient suffering and distress for families and clinicians. ${ }^{1,11,14}$

This study aimed to describe the medications used in MAiD in Ontario and British Columbia, their impact on time until death and complication rates in order to optimize the technical aspects of providing MAiD.

\section{Methods}

\section{Study design and setting}

This was a retrospective cohort study using data from institutional databases and chart review. The cohort was composed from 2 data sources: patients who received MAiD in Ontario between 2016 and 2018, and patients who received MAiD in 1 of 3 Canadian academic hospitals (in Hamilton and Ottawa, Ontario, and Vancouver, British Colombia) between 2019 and 2020 .

\section{Data sources}

We used de-identified data from the Office of the Chief Coroner (OCC) for Ontario MAiD Database, which includes information regarding all Ontarians who have legally died with medical assistance from June 2016 onward. The database is updated by nurse investigators, who receive copies of paper documentation and speak to the physician who provided MAiD and a family member for verification. As reporting to the OCC for Ontario is required by Section 10.1 of the Ontario Coroner's Act ${ }^{15}$ the number of unreported MAiD deaths is likely very small; this has been confirmed by means of cross-validation with pharmacy reports. ${ }^{10}$

Because the timing of medication administration was recorded in the OCC for Ontario MAID Database only up to the end of 2018, we used data from records at the investigators' institutions (Hamilton Health Sciences, The Ottawa Hospital and Vancouver Coastal Health) to determine the timing of intravenous drug administration from 2019 to 2020. We collected data from these sources on a spreadsheet template identical to that used by the OCC for Ontario for mandatory reporting. In doing so, we created the largest data set we could access that had the relevant information on drug dosages and timing of administration.

\section{Population}

All adult (age $\geq 18$ yr) patients who received MAiD in Ontario between 2016 and 2018, and in the 3 academic hospitals between 2019 and 2020 were included in the study. We collected data on patient characteristics (age, sex and diagnosis), the location of MAiD provision, type of MAiD provider (nurse practitioner or physician [and specialty, where available]), medications and dosages used, and complications. We grouped the location of MAiD into 3 broad categories: hospital, hospice or palliative care facility, or community or other (defined as private residence, retirement home, long-term care or complex care centre). Data on location of MAiD provision and physician specialty were not available for patients from Vancouver. We excluded participants for whom complete data on medication dosage and timing were unavailable and those in whom orally administered medications were used for MAiD. All chart review data were extracted by a single investigator at each site (Mic.K., S.O., L.W.) and entered into the spreadsheet. The data were cleaned and checked for accuracy by the analysis team (S.O., I.S., O.R.O.).

\section{Outcomes}

The primary outcome measure was length of time until death, as achieving a painless rapid death is a primary objective of MAiD. We defined time until death as the time from administration of the first medication recorded in the MAID procedure until death, as reported by the clinician providing MAiD. Secondary outcomes included factors associated with complications of MAiD, defined as need for a secondary MAiD kit, pain or burning on administration, or loss of intravenous access.

\section{Statistical analysis}

We summarized binary data as counts and percentages, and continuous variables with mean and standard deviation, or median and interquartile range (IQR), as appropriate. We conducted univariate analysis using the log-rank test to identify factors that had a significant association with survival time. We used a multilevel mixed-effects parametric survival model with Weibull distribution for multivariate analysis to account for the hierarchal structure of the data. We built the multivariable model using a combination of statistical metrics (likelihood ratio test) and clinical expertise. Variables included the administered medication types: premedication (typically midazolam), analgesic (typically lidocaine), anesthetic (propofol and phenobarbital), paralytic (rocuronium and cisatracurium) and cardiotoxic (bupivacaine and potassium chloride). Medications used were generally given at a few discrete standard dosages and were categorized based on median dosage into "low," "standard" and "high" dosages rather than treated as a continuous variable. The multivariable model was adjusted for patient age, patient sex and MAiD provider (nurse practitioner v. medical doctor). 
We conducted a complete case analysis and performed a post hoc sensitivity analysis excluding patients with time until death exceeding 1 hour, as we judged this to be exceedingly long and inclusion of these patients could affect the relation between medication and timing of death.

We analyzed the data using Stata, version 16 (StataCorp), with $p<0.05$ as the threshold for statistical significance. We did not calculate an a priori sample size as we used the entire sample population from all available sources.

\section{Ethics approval}

This study was reviewed and approved by the Hamilton Integrated Research Ethics Board (HIREB no. 7902).

\section{Results}

We analyzed the cases of 3557 adult patients (1786 men [50.2\%] and 1770 women [49.8\%] with a mean age of $74 \mathrm{yr}$ [standard deviation $13 \mathrm{yr}$ ], range 22-105 yr) who received MAiD between 2016 and 2020 (Figure 1, Table 1). Of the 3357,3113 (87.5\%) were from Ontario and 444 (12.5\%) were from Vancouver. Cancer was the most prevalent primary diagnosis (2519 patients [70.8\%]), followed by cardiovascular or respiratory disease $(840[23.6 \%])$. Most of the Ontario patients received MAiD in the community (1537 [49.4\%]) or in hospital (1382 [44.4\%]).

\section{Medications and dosages}

The medications and dosages used to perform MAiD are summarized in Table 2. Midazolam was the most common premedication sedative used (3251 patients [91.4\%]), with a median dosage of $10 \mathrm{mg}$ (IQR $10 \mathrm{mg}$ ) (range 1-70 mg). A total of 2477 patients $(69.6 \%)$ received the standard dosage of lidocaine as an analgesic (median dosage $40 \mathrm{mg}$ [IQR $20 \mathrm{mg}$ ], range $2-1000 \mathrm{mg})$. Twenty-one patients $(0.6 \%)$ received an opioid (morphine, hydromorphone or fentanyl). Almost all patients (3504 [98.5\%]) received propofol as an anesthetic, of whom 2999 (85.6\%) received the standard dosage of $1000 \mathrm{mg}$ (median dosage $1000 \mathrm{mg}$ [IQR $0 \mathrm{mg}$ ], range 1-3000 mg).

Rocuronium was the most commonly used paralytic (3228 patients [90.8\%]), with a median dosage of $200 \mathrm{mg}$ (IQR $0 \mathrm{mg}$ ) (range 10-400 mg); almost all patients (252/258 [97.7\%]) who received cisatracurium received a dosage of $30-40 \mathrm{mg}$. About one-quarter of patients (863 [24.3\%]) received cardiotoxic medications, most commonly bupivacaine (722 [83.7\%]) (median dosage $400 \mathrm{mg}$ [IQR $0 \mathrm{mg}$ ], range 20-2000 mg); the remaining 141 patients $(16.3 \%)$ received potassium chloride (median dosage $80 \mathrm{mEq}$ [IQR $0 \mathrm{mEq}$ ], range 10-1000 mEq).

\section{Time to death}

The median length of time from initiation of MAiD until death was 9 (IQR 6) minutes. The shortest documented time until death was 1 minute, and the longest documented time was 127 minutes.

In univariate analysis, we used Kaplan-Meier survival curves for each medication used in MAiD to explore which medications were associated with longer time until death. The median survival time increased from 9 to 12 minutes when patients received high-dose propofol rather than the standard dosage $(p<0.001)$ (Figure 2). Patients who received low and standard dosages of lidocaine had median survival times of 9 and 10 minutes, respectively, compared to 8 minutes for those who did not receive lidocaine (Appendix 1, Figure S1, available at www.cmajopen.ca/ content/10/1/E19/suppl/DC1). The difference in median time until death between patients who did not receive bupivacaine and those who received low, standard or high dosages was 1 minute. Patients who received a standard dosage of potassium chloride had a median survival time 2 minutes less than that of patients who did not receive potassium chloride. Compared to patients who did not receive midazolam, the median time until death was increased by 4 minutes, 2 minutes and 1 minute in those who received low-, standard- and high-dose midazolam, respectively.

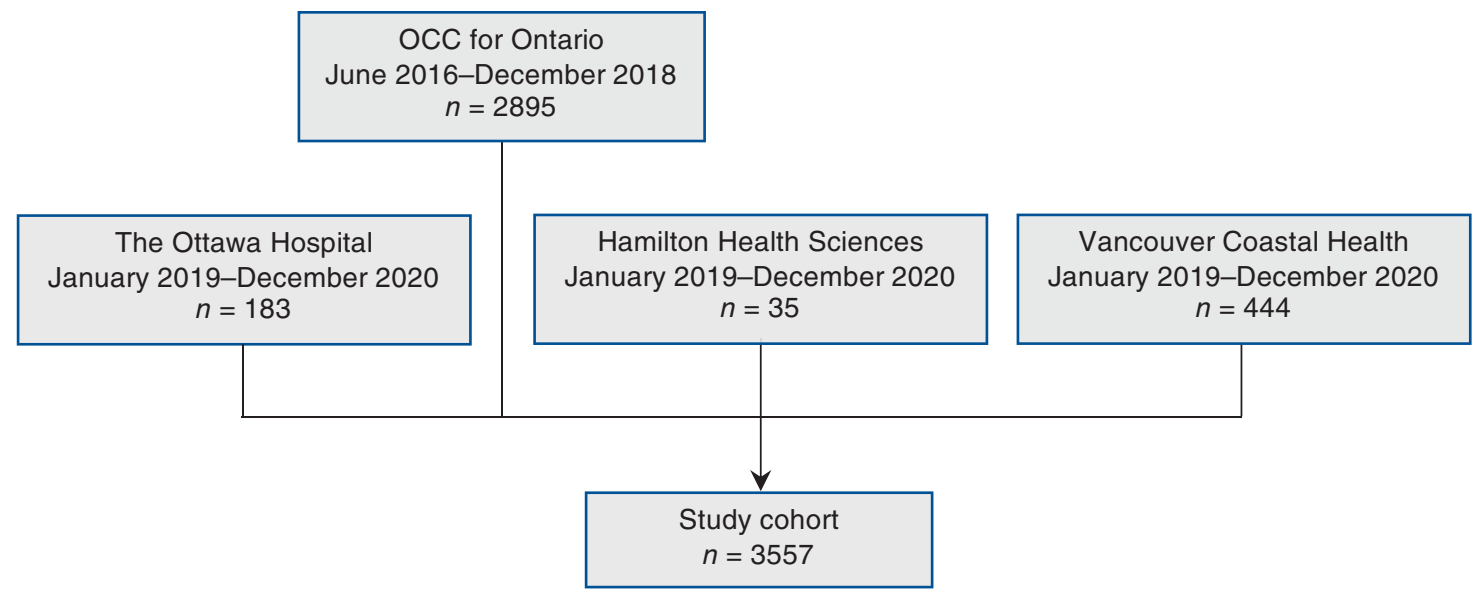

Figure 1: Flow diagram showing hierarchal structure of data sources. Note: $\mathrm{OCC}=$ Office of the Chief Coroner. 


\begin{tabular}{|c|c|}
\hline \multicolumn{2}{|c|}{$\begin{array}{l}\text { Table 1: Characteristics of intravenous provision of medical } \\
\text { assistance in dying in Ontario between } 2016 \text { and } 2018 \text { ( } n= \\
\text { 2895), and in } 3 \text { Canadian academic hospitals (in Hamilton } \\
\text { and Ottawa, Ontario, and Vancouver, British Columbia) } \\
\text { between } 2019 \text { and } 2020 \text { ( } n=662 \text { ) }\end{array}$} \\
\hline Characteristic & $\begin{array}{l}\text { No. }(\%) \text { of } \\
\text { patients* } \\
n=3557\end{array}$ \\
\hline Patient age, mean $\pm S D$, yr & $74 \pm 13.0$ \\
\hline \multicolumn{2}{|l|}{ Patient sex } \\
\hline Male & $1786(50.2)$ \\
\hline Female & $1770(49.8)$ \\
\hline Missing & $1(0.03)$ \\
\hline \multicolumn{2}{|l|}{ Location of MAiD provision $(n=3113) \dagger$} \\
\hline Hospital & $1382(44.4)$ \\
\hline Hospice or palliative care facility & $187(6.0)$ \\
\hline $\begin{array}{l}\text { Community/other (e.g., private residence, } \\
\text { long-term care) }\end{array}$ & $1537(49.4)$ \\
\hline Missing & $7(0.2)$ \\
\hline \multicolumn{2}{|l|}{ Patient diagnosis } \\
\hline Cancer & $2519(70.8)$ \\
\hline Neurologic disorder & $23(0.6)$ \\
\hline Cardiovascular/respiratory disorder & $840(23.6)$ \\
\hline Other & $126(3.5)$ \\
\hline Missing & $49(1.4)$ \\
\hline \multicolumn{2}{|l|}{ Provider profession } \\
\hline Physician & 3304 (92.9) \\
\hline Nurse practitioner & $240(6.7)$ \\
\hline Missing & $13(0.4)$ \\
\hline \multicolumn{2}{|l|}{ Physician specialty $(n=3113) \dagger$} \\
\hline Anesthesia & $473(15.2)$ \\
\hline Critical care & $95(3.0)$ \\
\hline Emergency medicine & $96(3.1)$ \\
\hline Family medicine & $1391(44.7)$ \\
\hline Internal medicine & $290(9.3)$ \\
\hline Neurology & $12(0.4)$ \\
\hline Oncology & $1(0.03)$ \\
\hline Palliative care & $382(12.3)$ \\
\hline Radiation oncology & $31(1.0)$ \\
\hline Surgery & 47 (1.5) \\
\hline Other & $10(0.3)$ \\
\hline Missing & $285(9.2)$ \\
\hline $\begin{array}{l}\text { Note: } \mathrm{MAiD}=\text { medical assistance in dying, } \mathrm{SD}=\text { stande } \\
\text { "Except where noted otherwise. } \\
\text { †For Ontario patients only. }\end{array}$ & ation. \\
\hline
\end{tabular}

When we plotted the Kaplan-Meier survival estimates against the log of time, they followed a linear trend, which indicated that the Weibull model was appropriate for the data. Results from the multivariate analysis are reported in Table 3.
After adjustment for the other medications, there was a statistically significant increased length of time from medication administration until death with high-dose propofol (hazard ratio [HR] $0.4,95 \%$ confidence interval [CI] 0.3-0.7). In multivariate analysis, administration of lidocaine was associated with prolonged time until death compared to no lidocaine (low-dose lidocaine: HR 0.8, 95\% CI 0.6-1.0; standard-dose: HR 0.6, 95\% CI 0.6-0.7; high-dose: HR 0.8, 95\% CI 0.7-0.9). The standard dosages of rocuronium and cisatracurium were associated with the same effect on time until death. Compared to no bupivacaine, the standard dosage of bupivacaine was associated with shorter time until death (HR 1.2, 95\% CI 1.01.3). The standard dosage of potassium chloride was not associated with shorter time until death (HR 1.2, 95\% CI 0.9-1.4).

\section{Sensitivity analysis}

A survival analysis excluding patients with time until death longer than 1 hour was conducted ( $n=2566$ analyzed). There was no difference in the associations between time until death and dosages used except for potassium chloride: after we excluded patients with greater than 1 hour from first injection until death, the relation was statistically significant (HR 1.5, 95\% CI 1.2-1.9).

\section{Complications}

There were 41 complications reported (1.2\% of cases), most of which fell into 1 of 2 main categories: problems obtaining intravenous access or loss of intravenous access after the MAiD procedure was started (23 cases), and prolonged time to death, necessitating a second kit (16 cases). The reasons for use of a second kit (e.g., intravenous access failure, drug underdosing, accidental wastage or breakage of medication) were not reported. The remaining 2 complications were pain on injection. There were no reported complications related to the specific medications used in the provision of MAiD. There was an insufficient number of complications to assess association with provider type or setting of MAiD.

\section{Interpretation}

Our study evaluated medications, dosages and complications of MAiD provided in Ontario from 2016 to 2020 and in Vancouver from 2019 to 2020 . The study population is similar to that described across Canada and elsewhere, with an older population, roughly equal sex balance, and cancer as the most prevalent diagnosis. ${ }^{16,17}$ The medications used for MAiD exhibited variability but also were in many ways homogenous, with midazolam, lidocaine, propofol and rocuronium being used far more often than any alternatives. Cardiotoxic agents were used in only a minority of patients. We identified associations between time from procedure start until death and medications. High-dose propofol and rocuronium were counterintuitively associated with a longer time until death, as was lidocaine. There was an association between use of potassium chloride and use of bupivacaine and a shorter time until death. The relation between bupivacaine and time to death was statistically significant in both 


\begin{tabular}{|c|c|c|c|c|c|c|c|}
\hline \multirow[b]{2}{*}{ Medication, unit } & \multirow{2}{*}{$\begin{array}{l}\text { No. (\%) of } \\
\text { patients }\end{array}$} & \multirow{2}{*}{$\begin{array}{l}\text { Median dosage } \\
(\mathrm{Q} 1, \mathrm{Q} 3 \text {, max })\end{array}$} & \multicolumn{5}{|c|}{$\begin{array}{l}\text { Dosage range; no. (\%) of patients } \\
\qquad n=3557\end{array}$} \\
\hline & & & High & Standard & Low & Missing & None \\
\hline Sedative & $3255(91.5)$ & & & & & & \\
\hline Midazolam, milligrams & $3251(91.4)$ & $\begin{array}{c}10 \\
(10,20,70)\end{array}$ & $\begin{array}{c}>19 \\
816(22.9)\end{array}$ & $\begin{array}{c}10-19 \\
2247(63.2)\end{array}$ & $\begin{array}{c}1-9 \\
188(5.3)\end{array}$ & $0 \overline{(0.0)}$ & $\begin{array}{c}0 \\
306(8.6)\end{array}$ \\
\hline Metoclopramide, $10 \mathrm{mg}$ & $4(0.1)$ & - & - & - & - & & - \\
\hline Analgesic & $2928(82.3)$ & & & & & & \\
\hline Lidocaine, milligrams & $2906(81.7)$ & $\begin{array}{c}40 \\
(40,60,1000)\end{array}$ & $\begin{array}{c}>60 \\
341(9.6)\end{array}$ & $\begin{array}{c}40-60 \\
2477(69.6)\end{array}$ & $\begin{array}{c}1-39 \\
88(2.5)\end{array}$ & $\begin{array}{c}- \\
0(0.0)\end{array}$ & $\begin{array}{c}0 \\
651(18.3)\end{array}$ \\
\hline $\begin{array}{l}\text { Magnesium sulfate, } \\
\text { milligrams }\end{array}$ & $1(0.0)$ & 5000 & - & - & - & & - \\
\hline Opioids (various) & $21(0.6)$ & & - & - & - & & - \\
\hline Anesthetic & 3527 (99.2) & & & & & & \\
\hline Propofol, milligrams & $3523(99.0)$ & $\begin{array}{c}1000 \\
(1000,1000,3000)\end{array}$ & $\begin{array}{l}>1000 \\
96(2.7)\end{array}$ & $\begin{array}{c}1000 \\
2999(84.3)\end{array}$ & $\begin{array}{c}1-999 \\
409(11.5)\end{array}$ & $19 \overline{(0.5)}$ & $\begin{array}{c}0 \\
34(1.0)\end{array}$ \\
\hline Phenobarbital, milligrams & $4(0.1)$ & $\begin{array}{c}3000 \\
(3000,3000,3000)\end{array}$ & - & - & - & & - \\
\hline Paralytic & $3486(98.0)$ & & & & & & \\
\hline Rocuronium, milligrams & $3295(92.6)$ & $\begin{array}{c}200 \\
(200,200,400)\end{array}$ & $\begin{array}{c}>200 \\
23(0.6)\end{array}$ & $\begin{array}{c}150-200 \\
2832(79.6)\end{array}$ & $\begin{array}{c}1-149 \\
373(10.5)\end{array}$ & $67 \overline{(1.9)}$ & $\begin{array}{c}0 \\
262(7.4)\end{array}$ \\
\hline Cisatracurium, milligrams & $325(9.1)$ & $\begin{array}{c}40 \\
(30,40,80)\end{array}$ & $\begin{array}{l}>40 \\
4(0.1)\end{array}$ & $\begin{array}{c}30-40 \\
252(7.1)\end{array}$ & $\begin{array}{c}1-29 \\
2(0.1)\end{array}$ & $67 \overline{(1.9)}$ & $\begin{array}{c}0 \\
3232(90.9)\end{array}$ \\
\hline Cardiotoxic medication & $863(24.3)$ & & & & & & \\
\hline Bupivacaine, milligrams & $722(20.3)$ & $\begin{array}{c}400 \\
(400,400,2000)\end{array}$ & $\begin{array}{c}>400 \\
18(0.5)\end{array}$ & $\begin{array}{c}400 \\
582(16.4)\end{array}$ & $\begin{array}{c}1-399 \\
122(3.4)\end{array}$ & $0 \overline{(0.0)}$ & $\begin{array}{c}0 \\
2835(79.7)\end{array}$ \\
\hline $\begin{array}{l}\text { Potassium chloride, } \\
\text { milliequivalents }\end{array}$ & $141(4.0)$ & $\begin{array}{c}80 \\
(80,80,1000)\end{array}$ & $\begin{array}{l}>80 \\
7(0.2)\end{array}$ & $\begin{array}{c}80 \\
129(3.6)\end{array}$ & $\begin{array}{c}1-79 \\
5(0.1)\end{array}$ & $0 \overline{(0.0)}$ & $\begin{array}{c}0 \\
3416(96.0)\end{array}$ \\
\hline
\end{tabular}

the primary and sensitivity analyses. Reports of complications were rare and were mainly related to intravenous access.

The observed associations between time from procedure start to death and medications suggests that the MAiD protocol used affects outcomes. The clinical relevance of differences in protocols - most affecting time to death by only a few minutes on average - to patient comfort and family satisfaction is unclear, although as patients are unconscious almost immediately after the procedure is started, the impact is likely greatest on families and clinicians. There is no clear definition of "clinically significant" prolongation of a MAiD procedure. However, in the present study, death occurred within 3-15 minutes in most cases, and it is reasonable to postulate that deaths that take much longer than this may cause distress for families and clinicians, who will worry about the possibility of a technical failure such as intravenous access failure or underuse of medications. Thus, although on average, these differences may be minor, in aggregate they may substantially affect patient, family and clinician experiences during the MAiD procedure; more research is needed to explore this relation. In situations in which a very short time from procedure start until death is important (e.g., organ donation after MAiD) or for patients who are expected to have a prolonged death owing to physiologic robustness, it seems sensible to choose a medication protocol that is least likely to result in prolonged time to death.

The observed association between administration of highdose propofol or rocuronium and longer time until death may have been due to use of higher dosages of these medications in cases in which an initial injection was thought to be inadequate, owing to reduced effectiveness or concerns about intravenous line placement. It is also possible that the prolonged time to death with high-dose propofol was due to unreported or unrecognized technical complications, as when clinicians used a backup kit, they may not have reported it as a complication. Alternatively, the higher dosage of medication may take longer to inject and thus increase the duration of the procedure, or clinicians concerned about discomfort may have administered lidocaine and infused propofol more slowly. In the case of lidocaine, the longer time to death may have been due to the antiarrhythmic effects of the drug, which may prolong the time until cardiac arrest. ${ }^{18}$ The low frequency of pain on injection was owing to either underreporting or low incidence. If the latter, this suggests that lidocaine may not be necessary and contributes to a potentially unnecessarily longer time until death. 


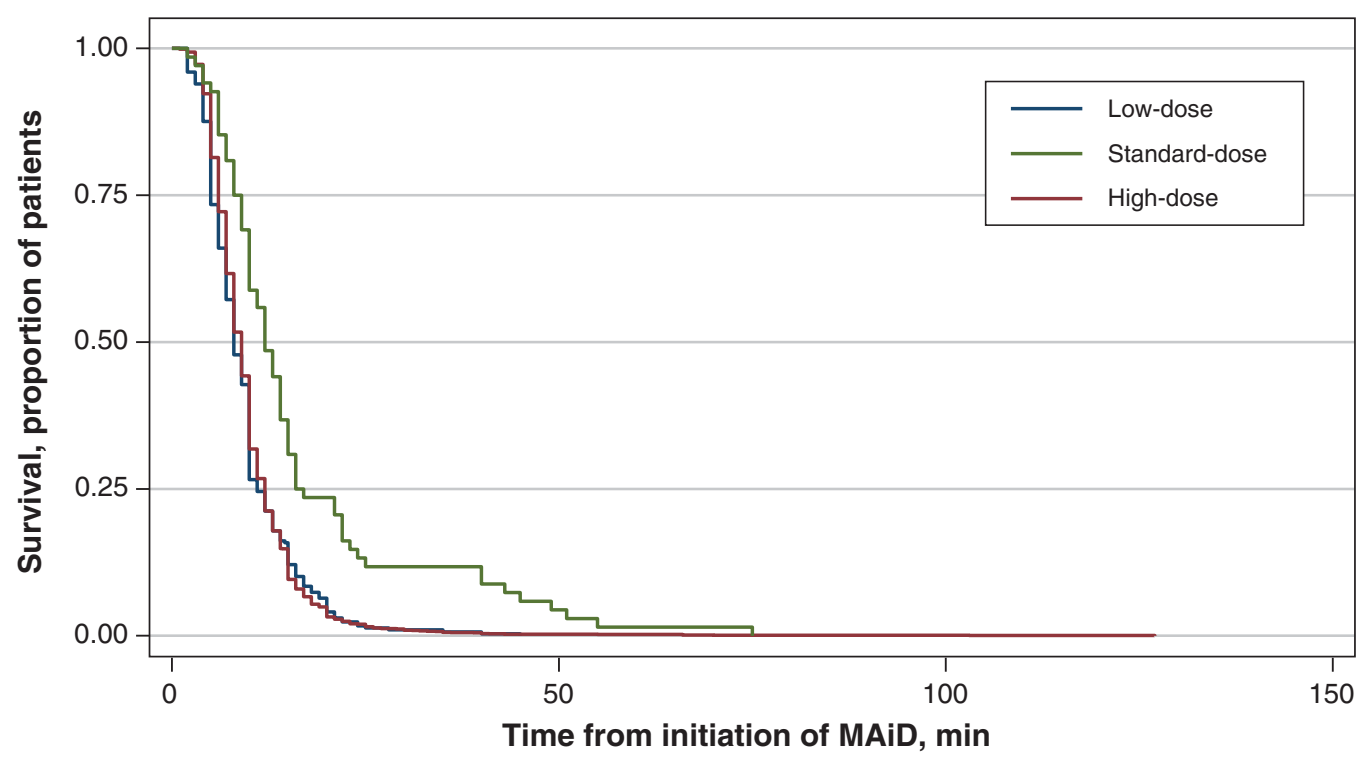

Figure 2: Kaplan-Meier survival curve comparing low-, standard- and high-dose propofol. Note: MAiD = medical assistance in dying.

Although cardiotoxic agents were used in a minority of cases, it appears their use is associated with hastened death. Potassium chloride decreases the membrane resting potential of cardiac cells, thus preventing myocardial repolarization, and bupivacaine blocks sodium channels throughout the heart and leads to acute conduction disturbances. ${ }^{19}$

Identifying patients in whom vascular access may be difficult before initiation of MAiD and acquiring the most skilled providers to insert the intravenous line may minimize unnecessary discomfort. As the data set we used was repurposed for analysis, it is possible that other technical complications (e.g., patient discomfort, seizures, anaphylaxis) were not reported or captured in this analysis. The OCC for Ontario routinely speaks with patients' families after a MAiD death. Thus, any major complications unrecognized by clinicians may be reported by families, and these would have been captured in the data set. Therefore, it is reassuring that complications were uncommon or were insufficiently troubling to clinicians, patients and families to even be recognized as such.

A complication that may not have been captured is the possibility of consciousness during the MAiD procedure, a complication also not reported in recent reviews. ${ }^{1,20}$ Most anesthetic dosages given during MAiD are greater than those necessary for major surgery; however, variations in dosing regimens, as reported here, raise the possibility that some patients may receive subtherapeutic dosages of anesthetic agents, resulting in awareness during the procedure, which would be difficult to assess. For this reason, we encourage the administration of fixed (rather than titrated) dosages of anesthetic agents, reviewed by pharmacists to ensure sufficient therapeutic efficacy, and diligent clinician assessment and documentation of coma induction. ${ }^{21}$
The observed variability in medications used for MAiD was expected based on a previous review ${ }^{1}$ and a Canadian Association of MAiD Assessors and Providers guidance document. ${ }^{12}$ Vander Stichele and colleagues ${ }^{21}$ evaluated the drugs used for euthanasia in Belgium by contacting a random sample of clinicians in 1998; detailed drug data were provided in 17 cases. A wider variety of medications than was used in our study was reported, including opioids, benzodiazepines, insulin, potassium chloride, lidocaine, neuromuscular blockers and barbiturates, given alone or in combination. The time from administration to death was reported in 11 cases and ranged from 4 minutes to more than 900 minutes; it was generally much longer than that seen with modern MAiD protocols, which emphasizes the value and predictability of the modern practice of overadministration of ad hoc medications. In a repeated population-based mortality follow-back study conducted from 1998 to 2013, the use of recommended drugs (barbiturate followed by neuromuscular blocker) increased, and the use of nonrecommended drugs (benzodiazepines, opioids without the recommended barbiturate and neuromuscular blockers) decreased, ${ }^{22}$ which suggests increased standardization of practice.

Horikx and Admiraal ${ }^{23}$ found similar results in a Dutch study conducted in 1998-2000 that included 154 patients. The medications used were varied (combinations of opioids, benzodiazepines and barbiturates followed by neuromuscular blockers), and the time to death ranged from 0 minutes (i.e., during injection of the first agent, in all cases thiopental) to 45 minutes. Again, these variations, both in medications used and in time until death, were much larger than those seen in our study. Findings of shorter procedure times from another study from the Netherlands also suggest increasing standardization of practice. ${ }^{13} \mathrm{~A}$ barbiturate followed by a 


\begin{tabular}{|c|c|c|}
\hline \multicolumn{3}{|c|}{ Table 3: Multivariable adjusted hazard ratios for time to death } \\
\hline Medication/category & $\begin{array}{l}\text { Median time } \\
\text { until death } \\
(\mathrm{Q} 1, \mathrm{Q} 3), \mathrm{min}^{*}\end{array}$ & $\mathrm{HR}(95 \% \mathrm{Cl})$ \\
\hline Lidocaine $($ Ref = none) & $8(6,10)$ & \\
\hline Low-dose & $6(7,12)$ & $0.8(0.6-1.0)$ \\
\hline Standard-dose & $9(6,12)$ & $0.6(0.6-0.7)$ \\
\hline High-dose & $8(6,10)$ & $0.8(0.7-0.9)$ \\
\hline Propofol (Ref = low-dose) & $8(5,11)$ & \\
\hline None & $7(6,15)$ & $1.4(0.5-1.0)$ \\
\hline Standard-dose & $9(6,12)$ & $0.9(0.8-1.0)$ \\
\hline High-dose & $12(8,16)$ & $0.4(0.3-0.5)$ \\
\hline $\begin{array}{l}\text { Paralytic }(\text { Ref }=\text { standard- } \\
\text { dose rocuronium })\end{array}$ & $9(6,12)$ & \\
\hline \multicolumn{3}{|l|}{ Rocuronium } \\
\hline Low-dose & $9(6,11)$ & $0.9(0.8-1.0)$ \\
\hline High-dose & $14(8,22)$ & $0.4(0.3-0.7)$ \\
\hline \multicolumn{3}{|l|}{ Cisatracurium } \\
\hline Standard-dose & $9(12,13)$ & $1.0(0.9-1.0)$ \\
\hline Low-dose & $14 \dagger$ & $0.8(0.1-5.6)$ \\
\hline High-dose & $17(14,43)$ & $0.4(0.1-1.3)$ \\
\hline Bupivacaine (Ref = none) & $9(6,12)$ & \\
\hline Low-dose & $8(5,12)$ & $1.0(0.8-1.3)$ \\
\hline Standard-dose & $8(5,12)$ & $1.2(1.0-1.3)$ \\
\hline High-dose & $8(6,20)$ & $0.7(0.4-1.2)$ \\
\hline $\begin{array}{l}\text { Potassium chloride } \\
\text { (Ref = none) }\end{array}$ & $9(6,12)$ & \\
\hline Low-dose & $10(8,13)$ & $1.3(0.5-3.5)$ \\
\hline Standard-dose & $7(5,9)$ & $1.2(0.9-1.4)$ \\
\hline High-dose & $14(11,15)$ & $0.7(0.3-1.6)$ \\
\hline \multicolumn{3}{|c|}{$\begin{array}{l}\text { Note: } \mathrm{Cl}=\text { confidence interval, } \mathrm{HR}=\text { hazard ratio, } \mathrm{Ref}=\text { reference category. } \\
\text { *Unadjusted for covariates. } \\
\text { †One patient. }\end{array}$} \\
\hline
\end{tabular}

neuromuscular blocker was used in most cases, with a correspondingly shorter median time from injection until death (10 min). Prolonged time to death (median $180 \mathrm{~min}$ ) occurred in only $10 \%$ of cases.

The present study expands on the limited existing literature on intravenous provision of MAiD and includes data more reflective of current practice, including the nearexclusive use of propofol over barbiturates, and a fairly small set of medications and dosages, potentially owing to adoption of guidelines and protocols. We did, however, note variations in practice that may be clinically relevant. In addition to guiding further research on MAiD pharmacology and helping clinicians choose medications to be used in MAiD, our findings may be useful in developing decision aids and educational materials for patients and families. Having knowledge about the medications, time until death and potential complications of MAiD may enable them to make more informed choices. ${ }^{24}$

\section{Limitations}

Because this is a retrospective study, unmeasured confounding limits any inferences of causation regarding the effects of the medications used in MAiD. For instance, clinicians may have given lower dosages to patients with a lack of cardiopulmonary reserve, or higher dosages to those with high medication tolerance due to prolonged opioid use. Although we adjusted for several variables for which data were available, it is possible that residual confounding accounts for some of the differences seen or, alternatively, may have mitigated real effects. Prospective research, including randomized controlled trials, is needed to further delineate relations between MAiD medications and clinical outcomes.

The clinical information was collected and repurposed for this study. As such, errors may have occurred during the data collection and extraction phases. The lack of standardization of data collection by clinicians across sites in the OCC for Ontario MAiD Database and at the additional study centres may have resulted in underreporting of complications, despite mandatory reporting requirements. Although we used the simple OCC for Ontario data collection spreadsheet to collect data at each site, this was done only by a single investigator, and errors may have occurred. It is also possible that some MAiD cases may have been unreported, but these numbers are likely small.

The demographic characteristics of patients in the study most closely relate to those of patients in Ontario, where the majority of the study data were obtained. The OCC for Ontario MAiD Database ceased collecting information on the time of administration at the end of 2018. We thus used supplemental data from 2019 onward, obtained from the investigators' centres, and although these data sets are small in comparison, combining them may have overemphasized the practice patterns at those centres. However, on the basis of physiology, the relations between medications, time to death and complications are unlikely to vary greatly between regions and are more likely to reflect differences in patient characteristics.

Given that this was a preliminary investigation, the clinical impact of our findings is uncertain. To evaluate the medications and dosages used to perform MAiD and the patient experience effectively, the outcomes evaluated must be defined as important to patients and their families. To our knowledge, there is no patient-reported outcome measure evaluating the technical quality of MAiD from the patient and family perspective, although efforts are ongoing. ${ }^{25,26}$

\section{Conclusion}

There was variability in the medications used to provide MAiD in Ontario and Vancouver. These variations were associated with small but potentially important differences in time from procedure start until death. Further research is needed to measure these differences prospectively and more accurately, and to explore patient preferences, including the preferred length and setting for a "good death" in the context of MAiD, to ensure clinicians use medications that result in predictable outcomes that are consistent with patient and family expectations for assisted death. 


\section{References}

1. Zworth M, Saleh C, Ball I, et al. Provision of medical assistance in dying: a scoping review. BM7 Open 2020;10:e036054.

2. Keown J. Euthanasia, ethics and public policy: an argument against legalization. 2nd ed. Cambridge (UK): Cambridge University Press; 2018.

3. Regulations for the Monitoring of Medical Assistance in Dying (SOR/2018166). Ottawa: Government of Canada [Justice Laws website]. 2021 Sept. 11, amended 2019 Nov. 1 Available: https://laws-lois.justice.gc.ca/eng/regulations/ SOR-2018-166/index.html (accessed 2020 May 3).

4. Emanuel EJ, Onwuteaka-Philipsen BD, Urwin JW, et al. Attitudes and practices of euthanasia and physician-assisted suicide in the United States, Canada, and Europe. 7AMA 2016;316:79-90.

5. Nicol J. Medical assistance in dying: the law in selected jurisdictions outside Canada. Publication no 2015-116-E. Ottawa: Library of Parliament; 2015.

6. Quill TE. Legal regulation of physician-assisted death: the latest report cards. NEngl 7 Med 2007;356:1911-3.

7. Hedberg K, New C. Oregon's Death with Dignity Act: 20 years of experience to inform the debate. Ann Intern Med 2017;167:579-83

8. Bill C-14. Ottawa: Parliament of Canada; 2016. Available: https://www.parl. ca/DocumentViewer/en/42-1/bill/C-14/royal-assent (accessed 2020 May 3).

9. Health Canada. Second annual report on medical assistance in dying in Canada 2020. Ottawa: Government of Canada; 2021. Cat no H22-1/6E-PDF. Available: https:/www.canada.ca/en/health-canada/services/medical-assistance-dying/ annual-report-2020.html (accessed 2021 Dec. 17).

10. CFPC Task Force on End-of-Life Care. A guide for reflection on ethical issues concerning assisted suicide and voluntary euthanasia. Mississauga (ON): College of Family Physicians of Canada; 2015.

11. Ganzini L, Nelson HD, Schmidt TA, et al. Physicians' experiences with the Oregon Death with Dignity Act. N Engl 7 Med 2000;342:557-63.

12. Intravenous MAiD medication protocols in Canada: review and recommendations. Victoria: Canadian Association of MAiD Assessors and Providers; 2020.

13. Groenewoud JH, van der Heide A, Onwuteaka-Philipsen BD, et al. Clinical problems with the performance of euthanasia and physician-assisted suicide in the Netherlands. NEngl 7 Med 2000;342:551-6.

14. Hendin H, Rutenfrans C, Zylicz Z. Physician-assisted suicide and euthanasia in the Netherlands. Lessons from the Dutch. $7 A M A$ 1997;277:1720-2.

15. Coroners Act, RSO. 1990, c. C.37. Available: https://www.ontario.ca/laws/ statute/90c37 (accessed 2021 Dec. 17).

16. Wu JSY, Pinilla J, Watson M, et al. Medical assistance in dying for cancer patients one year after legalization: a collaborative approach at a comprehensive cancer centre. Curr Oncol 2018;25:e486-9.

17. Meier DE, Emmons CA, Litke A, et al. Characteristics of patients requesting and receiving physician-assisted death. Arch Intern Med 2003;163:1537-42.

18. Sanfilippo F, Corredor C, Santonocito C, et al. Amiodarone or lidocaine for cardiac arrest: a systematic review and meta-analysis. Resuscitation 2016;107:31-7.

19. Schmitt N, Grunnet M, Olesen SP. Cardiac potassium channel subtypes: new roles in repolarization and arrhythmia. Physiol Rev 2014;94:609-53.

20. Boivin A, Marcoux I, Garnon G, et al. Comparing end-of-life practices in different policy contexts: a scoping review. F Health Serv Res Policy 2015;20:115-23.

21. Vander Stichele RH, Bilsen JJ, Bernheim JL, et al. Drugs used for euthanasia in Flanders, Belgium. Pharmacoepidemiol Drug Saf 2004;13:89-95.

22. Dierickx S, Cohen J, Vander Stichele R, et al. Drugs used for euthanasia: a repeated population-based mortality follow-back study in Flanders, Belgium, 1998-2013. F Pain Symptom Manage 2018;56:551-9.
23. Horikx A, Admiraal PV. Utilization of euthanatic agents; experience of physicians with 227 patients, 1998-2000 [article in Dutch]. Ned Tijdschr Geneeskd 2000;144:2497-500

24. Boivin A, Gauvin FP, Garnon G, et al. Information needs of francophone health care professionals and the public with regard to medical assistance in dying in Quebec: a qualitative study. CMA7 Open 2019;7:E190-6.

25. Brown J, Goodridge D, Harrison A, et al. Medical assistance in dying: patients', families', and health care providers' perspectives on access and care delivery. 7 Palliat Med 2020;23:1468-77.

26. Hales BM, Bean S, Isenberg-Grzeda E, et al. Improving the medical assistance in dying (MAiD) process: a qualitative study of family caregiver perspectives. Palliat Support Care 2019;17:590-5.

Affiliations: Department of Medicine (Stukalin), University of Calgary, Calgary, Alta.; Division of Plastic Surgery (Olaiya), Department of Surgery, McMaster University, Hamilton, Ont.; The Ottawa Hospital (Naik, Kekewich, Wilding); Department of Anesthesiology and Pain Medicine (Naik), University of Ottawa, Ottawa, Ont.; Department of Family Practice (Wiebe), University of British Columbia, Vancouver, BC; London School of Hygiene and Tropical Medicine (Kelly), University of London, London, UK; Office of the Chief Coroner for Ontario (Halko), Toronto, Ont.; Departments of Medicine (Oczkowski), and Health Research Methods, Evidence, and Impact (Olaiya, Oczkowski), McMaster University, Hamilton, Ont.

Contributors: Igor Stukalin and Oluwatobi Olaiya share first authorship. Viren Naik, Ellen Wiebe, Mike Kekewich, Michaela Kelly and Simon Oczkowski conceived and designed the study. Igor Stukalin, Oluwatobi Olaiya and Simon Oczkowski drafted the manuscript. All of the authors acquired, analyzed and interpreted the data, revised the manuscript for important intellectual content, approved the final version to be published and agreed to be accountable for all aspects of the work.

Funding: Simon Oczkowski is supported by an internal career award from the Department of Medicine, McMaster University.

Content licence: This is an Open Access article distributed in accordance with the terms of the Creative Commons Attribution (CC BY-NCND 4.0) licence, which permits use, distribution and reproduction in any medium, provided that the original publication is properly cited, the use is noncommercial (i.e., research or educational use), and no modifications or adaptations are made. See: https://creativecommons.org/licenses/by-nc $-\mathrm{nd} / 4.0 \%$

Data sharing: No data are available. The Office of the Chief Coroner for Ontario MAiD Database is available to researchers on request but is not under the control or authority of the authors.

Supplemental information: For reviewer comments and the original submission of this manuscript, please see www.cmajopen.ca/content/10/ 1/E19/suppl/DC1. 